\title{
Nuclear Bud Formation: A Novel Manifestation of Zidovudine Genotoxicity
}

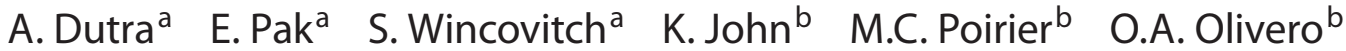 \\ ${ }^{a}$ Cytogenetics and Microscopy Core, National Human Genome Research Institute and ${ }^{b}$ Laboratory of Cancer \\ Biology and Genetics, Center for Cancer Research, National Cancer Institute, NIH, Bethesda, Md., USA
}

\section{Key Words}

Aneuploidy $\cdot$ AZT $\cdot \mathrm{FISH} \cdot$ Mouse painting probes $\cdot$

Nuclear buds $\cdot$ Zidovudine

\begin{abstract}
Normal diploid somatic mammalian cell division generates 2 daughter cells as a result of a strict and well-controlled mitotic process. However, some defects during the progression of that process could generate an unbalanced distribution of chromosomes, aneuploidy and eventually, a malignant phenotype. Previous observations using a transgenic mouse model with diminished DNA repair capacity revealed the presence of nuclear buds (NBs) induced in vitro by the nucleoside analog zidovudine (Retrovir ${ }^{\circledR}, 3^{\prime}$-azido-3'-deoxythymidine, AZT). Here we used bone marrow mesenchymal cells, taken from mice with the $X \mathrm{pa}^{-/-} \mathrm{Trp53^{+/- }}$ genotype, that were cultured and exposed to 0 and $100 \mu \mathrm{M}$ AZT for 24 hours. Fixed and denatured cells were processed by fluorescence in situ hybridization (FISH) with whole chromosome painting probes used to identify chromosomes in cells growing on glass chamber slides ( 2 probes/slide). A variety of sizes and shapes of NBs were observed. Some NBs had a large connection with the main nucleus ( $>1 / 4$ of the NB diameter), others had a smaller connection ( $<1 / 4$ of the NB diameter), some were circular and positioned close to the nucleus, while some resided in the cytoplasm separated from the nucleus or connected by a thin chromatin strand. We had hypothe-
\end{abstract}

sized that NBs would progress in the process of budding until separation occurred, but this was not proven by timelapse photography studies performed for 20 hours. From 1,126 cells scored in the unexposed cultures, $10.39 \%$ of cells carried NBs, while from 1,108 cells scored in the AZT-exposed cultures $29.16 \%$ of cells carried NBs ( $p=0.001$ ). In AZT-exposed cells there were a total of 322 NBs scored; $46.6 \%$ or 150 NBs contained positive signals for one or both probes used, while $53 \%$ or 172 NBs had no probe signal. In addition, FISH analysis showed no preferential localization of any chromosome within the NBs. Among the NBs that carried no probe signal, the presence of positive signals with inversion of DAPI imaging demonstrated centromeric content. It has been hypothesized that NBs occur as a result of expulsion of amplified DNA from the main nucleus; however, this data demonstrates that NBs may contain any chromosome, suggesting that NBs do not consist of just amplified DNA.

Copyright $\odot 2010$ S. Karger AG, Basel

Normal diploid somatic mammalian cell division generates 2 daughter cells as a result of a strict and well-controlled mitotic process. However, some defects during the progression of that process could generate an unbalanced distribution of chromosomes, aneuploidy and eventually, a malignant phenotype. In that regard, expansion of the nucleus into structures designated as nuclear buds (NBs) has attracted the attention of many researchers. NBs have

\section{KARGER}

(C) 2010 S. Karger AG, Basel

Fax +41613061234

E-Mail karger@karger.ch

www.karger.com
Accessible online at: www.karger.com/cgr
O.A. Olivero

National Cancer Institute, NIH, Laboratory of Cancer Biology and Genetics Carcinogen-DNA Interactions Section, 37 Convent Dr. MSC 4255, Bldg 37 Rm 4032 Bethesda, MD 20892-4255 (USA)

Tel. +1 301435 7843, Fax +1 301402 0153, E-Mail olivero@exchange.nih.gov 
been defined as structures that are responsible for the expulsion of undesirable DNA content, such as amplified DNA [Shimizu et al., 1998, 2000]. Some reports indicate that NBs, called 'blebs', are precursors of micronuclei and contain specific chromosomes, like chromosomes 1, 4 and 12 in human liposarcomas [Pedeutour et al., 1994; Suijkerbuijk et al., 1994], or chromosomes 17 and 21 in folate deficiency-induced aneuploidy in human lymphocytes [Wang et al., 2004].

The nucleoside analog drug zidovudine (Retrovir ${ }^{\circledR}$, 3'-azido-3'-deoxythymidine, AZT) is a DNA replication chain terminator able to induce genomic centrosome amplification and aneuploidy in a number of species [Borojerdi et al., 2009; Yu et al., 2009]. In addition, AZT has been shown to induce NBs in AZT-exposed cultured hamster cells [Borojerdi et al., 2009].

Here we expand those original observations and present experiments designed to identify the content of NBs induced in mesenchymal-derived cells grown from the bone marrow of mice null for the Xpa gene and haploinsufficient for $\operatorname{Tr} p 53$ [van Steeg et al., 2001; Nostrand et al., 2009]. Employing fluorescent in situ hybridization (FISH) techniques, individual chromosomes were identified by localization within NBs. Additionally, the process of formation of the NBs was monitored by time-lapse photography. These studies show that the content of the NBs is diverse, and the genesis of the NB remains to be understood.

\section{Materials and Methods}

\section{Transgenic Mouse Model}

Experiments have been performed utilizing C57 BL mice that are null for the nucleotide excision repair gene Xpa and heterozygous for the tumor suppressor gene Trp53. The nomenclature used in this manuscript to represent the genotype will be $\mathrm{Xpa}^{-/-}$ $\operatorname{Tr} p 53^{+/-}$. Animals were maintained and treated at the NCI Animal Facility under conditions specified by the Association for the Assessment and Accreditation of Laboratory Animal Care, in accordance with humane principles for laboratory animal care. Procedures were approved by the NCI Animal Care and Use Committee. The selection of this transgenic model is based on prior observations demonstrating an enhanced response to the genotoxicity induced by the nucleoside analog AZT. Mice of this genotype are susceptible perhaps due to the fact that the animals lack the first step in nucleotide excision repair, and the lack of Trp53 allows the cells to progress through the cell cycle regardless of the DNA damage.

\section{Cell Culture}

Bone marrow from $\mathrm{Xpa}^{-1-} \mathrm{Trp} 53^{+/-}$mice was collected from the femur under sterile conditions employing a disposable LODOSE $1 / 2$ cc U-100 Insulin Syringe with a permanently attached
$28 \mathrm{G} \times 1 / 2$ " needle (Becton Dickinson, Franklin Lakes, N.J.). The material was resuspended in RPMI media (ATCC, Manassas, Va.) with $10 \%$ fetal bovine serum (ATCC) and cells were plated on onechamber polystyrene vessel glass slides (BD Falcon, Bedford, Mass.). Cells remained undisturbed for $48 \mathrm{~h}$ to allow attachment. After that period, media was removed, and replaced with fresh media. Mesenchymal-derived cells with a typical fibroblast appearance were attached by that time. When cultures were semiconfluent, the media was removed and replaced by media containing either 0 or $100 \mu$ M AZT (Sigma, St. Louis, Mo.). After $24 \mathrm{~h}$ cells were washed with PBS and fixed with ice-cold methanol at $-20^{\circ} \mathrm{C}$ for $20 \mathrm{~min}$.

\section{Chromosome Identification by Fluorescent Painting Probes}

A single whole mouse chromosome paint system (Applied Spectral Imaging, Vista, Calif.) was used to identify 2 pairs of chromosomes per slide by simultaneously using Spectrum Orange probes for one pair and Spectrum Green probes for the second pair. Slides were incubated for $2 \mathrm{~min}$ at room temperature in $2 \times$ SSC $(0.3 \mathrm{M} \mathrm{NaCl}$ and $0.3 \mathrm{M}$ sodium citrate $)$ and then dehydrated sequentially in $70 \%, 80 \%$, and $90 \%$ ethanol. Chromosomal DNA was denatured in $70 \%$ formamide, $2 \times$ SSC, for $2 \mathrm{~min}$ at $72^{\circ} \mathrm{C}$ followed by dehydration in ethanol washes of $70 \%, 90 \%$, and $100 \%$. FISH was carried out as described by Pinkel et al. [1986] and Lichter et al. [1988] with minor modifications. On each slide $10 \mu l$ of labeled DNA was applied. Labeled DNA was denatured at $78^{\circ} \mathrm{C}$ for $10 \mathrm{~min}$ and then preannealed at $37^{\circ} \mathrm{C}$ for $30 \mathrm{~min}$. Slides were hybridized overnight at $37^{\circ} \mathrm{C}$. Post-hybridization washes were performed at $45^{\circ} \mathrm{C}$ as follows: (1) $50 \%$ formamide, $2 \times$ SSC, $15 \mathrm{~min}$, (2) $1 \times$ SSC, $10 \mathrm{~min}$, and (3) $0.1 \times$ SSC, $10 \mathrm{~min}$. Slides were counterstained and mounted with DAPI-Antifade $250 \mathrm{ng} / \mu \mathrm{l}$ (Vector, Burlingame, Calif.).

\section{Microscopy and Chromosomal Content Analysis}

FISH slides were imaged with a Zeiss Fluorescent Imager M1 upright microscope system (Carl Zeiss Inc, Thornwood, N.Y.) equipped with a Hamamatsu Digital Camera (Hamamatsu, Bridgewater, N.J.) and IPlab software (BioVision, Exton, Pa.). Approximately 100 cells per pair of painting probes were scored with the NB content being analyzed for the presence or absence of the specific pair of painting probes in cultured $\mathrm{Xpa}^{-/-} \mathrm{Tr} p 53^{+/-}$bone marrow mesenchymal derived cells either unexposed or exposed to $100 \mu \mathrm{M}$ AZT for $24 \mathrm{~h}$.

\section{Time-Lapse Imaging}

Mesenchymal fibroblasts obtained from $\mathrm{Xpa}^{-/-} \mathrm{Tr} p 53^{+/-}$mice were cultured in $35 \mathrm{~mm}$ glass bottom culture dishes (Mat-Tek Corporation, Ashland, Mass.) and grown until semi-confluence was reached. Cells were then treated with 0 and $100 \mu \mathrm{M}$ AZT and stained with Hoechst 33342 FluoroPure grade (Invitrogen, Eugene, Oreg.). Cells were placed inside an environmental chamber (enclosing most of the microscope) set to $37^{\circ} \mathrm{C}$. A small 6" $\times 4$ " $\times 1$ " transparent chamber was placed over the Mat-Tek dish in order to supply $5 \% \mathrm{CO}_{2}$ and humidity to the cells. All experiments were imaged for at least a minimum of 17 and maximum of $20 \mathrm{~h}$ with no more than 30 locations per dish. All images were collected using a wide-field Personal DeltaVision system (Applied Precision Inc., Issaquah, Wash.) mounted on an inverted Olympus IX71 microscope with an oil immersion PlanApo N 60×/1.42 objective lens. All images were acquired using a CoolSNAP ES2 
camera with a frame rate of 2, 4 and 8 min per acquisition, $2 \times 2$ binning and a $512 \times 512$ pixels imaging field. Z-stacks were collected (3 images per stack) using excitation 360/40 (10\%) and emission filter $457 / 50$ for all image sets. Videos of the live cells were created using Applied Precision's SoftWoRx software package version 4.0.0.

\section{Results}

\section{Incidence of NBs}

A summary of the incidence of NBs in cultured $X p a^{-1-} \operatorname{Tr} p 53^{+/-}$bone-marrow mesenchymal cells exposed to 0 or $100 \mu \mathrm{M} \mathrm{AZT}$ is presented in table 1 . The total number of cells scored for 0 and $100 \mu \mathrm{M}$ AZT is shown, along with cells with and without NBs. Percentages of NB-containing cells are indicated in table 1 (bottom row). A Student's $t$ test comparison of the NBs in 0 and $100 \mu \mathrm{M}$ AZT treatments showed statistical significance $(\mathrm{p}=0.001)$.

\section{FISH Analysis of Specific Chromosomal Content of NBs}

A representative NB is shown in figure 1A. A total of approximately 100 cells was analyzed per FISH experiment. From those, the content of the NBs was scored to identify the presence of one or both of the mouse painting probes hybridized on that particular slide. A representative example is shown in figure 1B. Table 2 shows the number of NBs positive or negative for the painting probe hybridized on each slide. The first column shows the chromosome pair probed on each slide together with the labeling dyes: Spectrum Orange (red) and Spectrum Green (green). The second column represents the total number of NBs that contain positive signals for the probes used on that FISH experiment. The last column indicates the total number of NBs not containing positive signals for the specific pair of probes used in that hybridization. It is important to mention that the NBs classified as not containing FISH signal did frequently contain chromosomes that were not hybridized on that particular slide. From a total of 322 nuclei analyzed, 172 (53.42\%) showed no positive signals, however DAPI staining revealed centromeric signals, suggesting chromosomal content inside the NBs (fig. 1G, K).

\section{Interpretation of NB Images by Fluorescence \\ Microscopy}

Careful scoring of FISH cells by fluorescence microscopy suggested that NBs could be classified in a hypothetically-sequential progression based on size and mag-
Table 1. Incidence of NBs in cultured $X p a^{-/-} \operatorname{Tr} p 53^{+/-}$bone marrow mesenchymal cells exposed to 0 or $100 \mu \mathrm{M}$ AZT for 24 hours

\begin{tabular}{lcc}
\hline & Unexposed & AZT Exposed \\
\hline Total cells & 1,126 & 1,108 \\
Without NB & 1,009 & 782 \\
With NB & 117 & 326 \\
$\%$ cells with NB & 10.39 & 29.16 \\
\hline
\end{tabular}

a Student's t test revealed a significant difference between NBs present in the AZT group compared to the unexposed group.

$\mathrm{p}=0.001$.

nitude of connection with the nucleus (compare fig. 1B, D, F). Some NBs had a large connection with the main nucleus ( $>1 / 4$ of the NB diameter) (fig. $1 \mathrm{D}, \mathrm{F})$, others had a smaller connection $(<1 / 4$ of the NB diameter) (fig. 1A, arrowhead), some were circular and positioned close to the nucleus (fig. 1F, G), while some resided in the cytoplasm separated from the nucleus (fig. $1 \mathrm{~K}$, arrowhead) or connected by a thin chromatin strand (fig. 1A, arrowhead, also see online supplementary movie 1; for all online supplementary material, see www. karger.com/doi/10.1159/000298794).

\section{Evaluation of NB Formation by Time-Lapse \\ Photography (Frames 1 and 2)}

We had hypothesized that cells could progress from NBs with a large connection to the separated NB, but this was not proven by time-lapse photography studies performed for 17-20 hours. Live-cell imaging techniques were performed with a time-lapse rate of 2,4 and $8 \mathrm{~min}$ utes per acquisition. Obtaining photos every 2 minutes induced cell death in 6-8 hours due to phototoxicity by overexposure from the UV lamp used to reveal Hoechst images. Acquisitions every 8 minutes were not fast enough to follow the nuclei and process of NB formation. We found that acquisitions every 4 minutes worked well, and used this interval to follow nuclear changes and NB formation for 5 independent experiments each with 17-20 hours duration. The sequential progression of NBs from the initial stage of formation to the separated state could not be confirmed when 50 cells/experiment were observed over a time period of 17-20 hours, with a collection rate of 2 minutes per acquisition. A sudden expansion of the nuclear content to the final size of the NB was observed in the majority of the cases. Occasionally, the NBs were separated from the nucleus but frequently they remained at- 

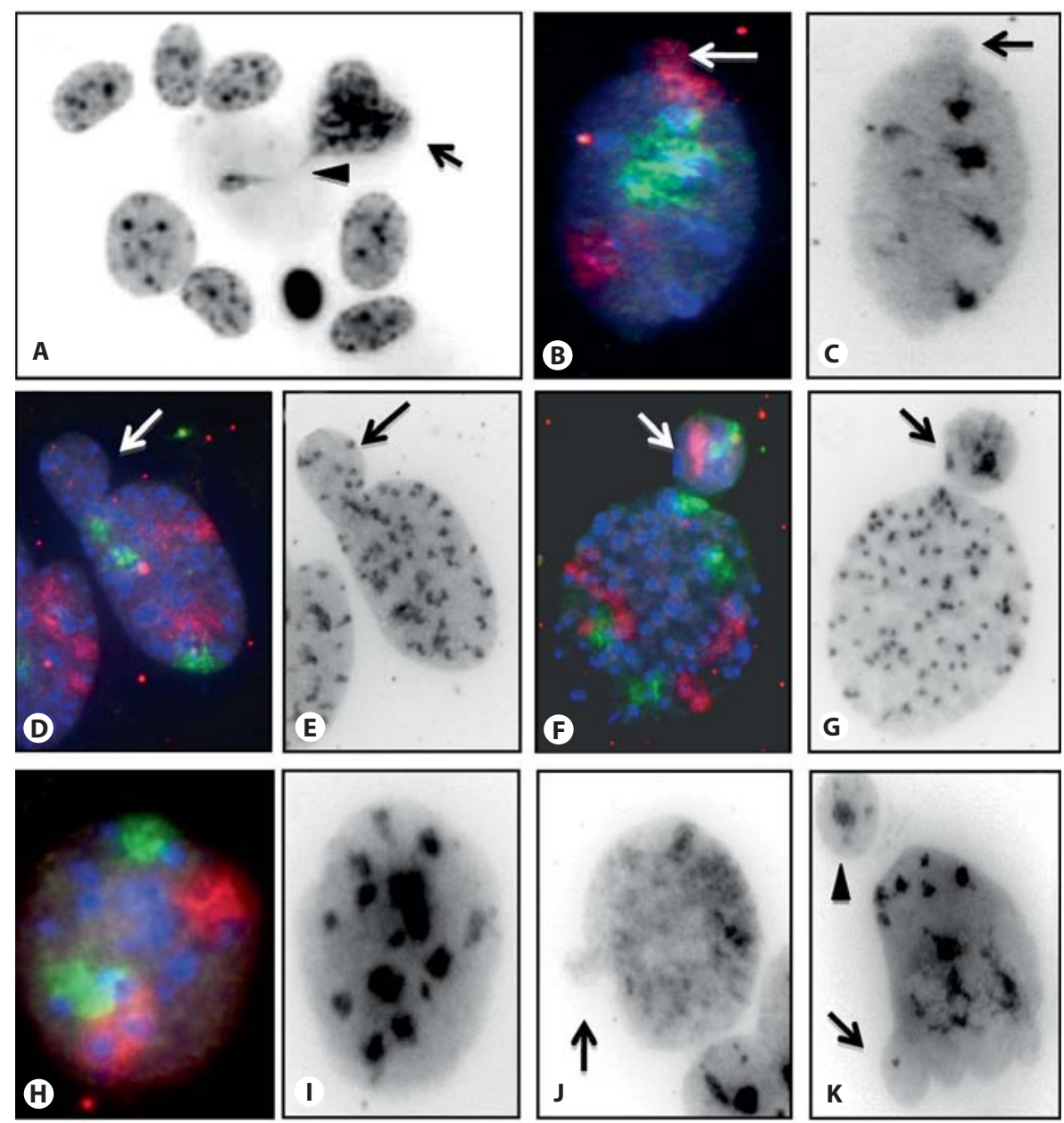

Fig. 1. NBs. A Frame obtained from time-lapse movie 1 showing inverted Hoechst image of nuclei of $\mathrm{Xpa}^{-/-} \mathrm{Tr} p 53^{+/-}$bone marrow mesenchymal derived cells exposed to $100 \mu \mathrm{M}$ AZT for 24 hours. A nucleus (arrow) exhibits an NB attached by a DNA strand (arrowhead). B, D, F, H Xpa $a^{-/-} \operatorname{Tr} p 53^{+/-}$mesenchymal-derived cells exposed to $100 \mu \mathrm{M}$ AZT hybridized with Spectrum Orange-labeled whole chromosome 1 and Spectrum Green-labeled whole chromosome 12 probes. B Nucleus bearing an NB in an initial state of formation containing part of chromosome 1 (arrow). C Inverted DAPI image of cell in (B) showing chromatin containing NB (arrow). D Nucleus with a larger NB showing the absence of both chromosomes 1 and 12 in the NB; $\mathbf{E}$ inverted DAPI image of cell in (D) showing NB-containing centromeres and chromatin

(arrow); F nucleus with NB containing labeled signal for both chromosomes 1 (red) and 12 (green); G inverted DAPI image of cell in $(\mathbf{F})$ showing centromeres and chromatin containing NB (arrow). H Normal nucleus showing typical localization of both Spectrum Orange-labeled whole chromosome 1 and Spectrum Green-labeled whole chromosome 12 probes. I Inverted DAPI image of nucleus in (H). J, K Inverted DAPI images of $\mathrm{Xpa}^{-/-} \mathrm{Tr} 553^{+/-}$ bone marrow mesenchymal derived nuclei from cells exposed to $100 \mu \mathrm{M}$ AZT for 24 hours showing different types of NBs. J NB containing no centromere staining (arrow); $\mathbf{K}$ nuclei with attached NB containing centromere (arrow), and a detached NB containing multiple centromeric signals (arrowhead).

tached by a thin strand of DNA (fig. 1A arrow and online suppl. video 1). The attached remote NBs and the nuclei kept the same degree of chromatin condensation, with synchronized progression of the condensation in the 2 independent bodies. The size of the NB depended on the ploidy of the cells, as larger NBs were more frequently associated with larger nuclei. Additionally, with time-lapse

imaging it was recognized that polyploid cells are more resistant to cellular death, than their diploid counterpart (online suppl. video 2).

\section{NB Content Is Variable}

At times whole chromosomes appeared to be located within an NB although the NB was not always positive 
for the painting probe (fig. 1D). Unstained (FISH negative) whole chromosomes can be identified by inverting the DAPI signal (fig. 1E, G, K, arrows). In many occasions $\mathrm{NBs}$ were positive for the painting probes, revealing the presence of specific chromosomes (fig. 1B, F) and in some cases the NBs were negative for any centromere or specific painting probe (fig. 1J, arrow). Figure 1B shows an NB containing part of chromosome 12 (arrow) and figure $1 \mathrm{~F}$ shows both chromosomes in a large NB (arrow). Specific chromosome content observed in a normal nucleus is shown in figure $1 \mathrm{H}$, where Spectrum Orange-labeled whole chromosome 1 and Spectrum Green-labeled whole chromosome 12 each exhibits 2 positive FISH signals.

\section{Discussion}

In previous studies the antiretroviral drug AZT has induced cell cycle arrest, micronuclei, sister chromatid exchanges, shortened telomeres, and alterations in gene expression [Olivero, 2007]. A novel form of DNA response to damage, described as budding of the nucleus, has been reported recently in $\mathrm{CHO}$ cells [Borojerdi et al., 2009]. Here we investigated the content of NBs, induced in cultured fibroblasts taken from transgenic mice with a DNA repair deficiency and a $\operatorname{Tr} p 53$ deficiency. Painting probes specific for mouse chromosomes were used to identify the presence/absence of whole chromosomes located within the NBs. According to Fenech's definition 'NBs are bodies of DNA attached to the nucleus by a narrow stalk of nucelopasmic material, depending on the stage of the budding process' [Fenech, 2007]. Although the time involved in the complete maturation of a bud is uncertain, some reports indicate that gamma irradiation induced NBs about 3 hours after the initial treatment [Haaf et al., 1999]. Although not specific, some evidence points towards the preferential material being expelled from the nucleus as unwound chromatin [Xu et al., 1999]. Furthermore, it has been proposed that the content of NBs originates from interstitial or terminal fragments, or excess DNA extruded from the nucleus [Lindberg et al., 2007]. Here we provide evidence that nuclear budding is a process that starts early after cells have been exposed to AZT, as shown in the time-lapse experiments obtained during a 17-20 hour period. Some NBs were observed as early as 2 hours after treatment was initiated. Analysis of content using whole chromosome painting probes revealed that AZT induces NBs in $~ 30 \%$ of cells while $10 \%$ of untreated cells have NBs. Around $47 \%$ of the NBs were
Table 2. FISH analysis of specific chromosomal content of NBs in $\mathrm{Xpa}^{-/-} \mathrm{Tr}_{\mathrm{p}} 53^{+/-}$cultured bone marrow mesenchymal cells exposed to $100 \mu \mathrm{M}$ AZT for 24 hours

\begin{tabular}{lll}
\hline Chromosome probe $^{\mathrm{a}}$ & $\begin{array}{l}\text { Cells with } \\
\text { NBs containing } \\
\text { FISH signal }\end{array}$ & $\begin{array}{l}\text { Cells with } \\
\text { NBs containing } \\
\text { no FISH signal }\end{array}$ \\
\hline 1 (red) +12 (green) & 11 & 16 \\
2 (red) + 13 (green) & 14 & 17 \\
3 (red) + 14 (green) & 9 & 12 \\
4 (red) + 15 (green) & 17 & 20 \\
5 (red) + 16 (green) & 17 & 19 \\
6 (red) + 17 (green) & 13 & 18 \\
7 (red) + 18 (green) & 12 & 15 \\
8 (red) + 19 (green) & 20 & 18 \\
9 (red) + X (green) & 5 & 6 \\
10 (red) + Y (green) & 19 & 21 \\
11 (red) & 13 & 10
\end{tabular}

a A total of 322 NBs were scored for 11 FISH experiments, 150 of those $(46.58 \%)$ were positive for FISH signals indicating the presence of either one or 2 chromosomes of the pair being analyzed, while 172 (53.42\%) show no FISH signal for the pair being hybridized.

positive for chromosome painting probes and $\mathrm{NB}$ content is not chromosome specific. DAPI-stained inverted images showed the presence of centromeric signals evidencing localization of whole chromosomes contained inside NBs. Nevertheless, time-lapse photography revealed that NBs: are dynamic structures that can change shape in space and time; can contain centromeres or DNA material; can remain attached to the main nucleus by a strand of DNA of variable size; maintain synchrony with the nucleus at least in terms of chromatin condensation; and, vary in size with ploidy of the cell, rather than the extent of DNA damage (i.e. larger nuclei produce larger NBs).

Live-cell imaging techniques were used to provide insights into the nature and function of NBs. Time-lapse photography experiments showed that NBs could be detached in several pieces from the original nucleus before reaching apoptosis. All the observations done in this study obtained by both FISH analysis and time-lapsed photography help to characterize NBs and clarify the genesis and fate of these structures in the life of the cell. We demonstrated that no specific chromosome is preferred to form NBs but most NBs show chromatin content. It remains to be understood, however, if there is a real function associated with expulsion of amplified DNA. 


\section{References}

Borojerdi JP, Ming J, Cooch C, Ward Y, SeminoMora C, et al: Centrosomal amplification and aneuploidy induced by the antiretroviral drug AZT in hamster and human cells. Mutat Res 665:67-74 (2009).

Fenech M: Cytokinesis-block micronucleus cytome assay. Nat Protoc 2:1084-1104 (2007)

-Haaf T, Raderschall E, Reddy G, Ward DC, Radding CM, Golub EI: Sequestration of mammalian Rad51-recombination protein into micronuclei. J Cell Biol 144:11-20 (1999).

Lichter P, Cremer T, Borden J, Manuelidis L, Ward DC: Delineation of individual human chromosomes in metaphase and interphase cells by in situ suppression hybridization us ing recombinant DNA libraries. Hum Genet 80:224-234 (1988).

Lindberg HK, Wang X, Jarventaus H, Falck GC, Norppa H, Fenech M: Origin of nuclear buds and micronuclei in normal and folate-deprived human lymphocytes. Mutat Res 617: 33-45 (2007).

Nostrand TA, Poirier MC, Olivero OA: Abnormal centrosomal amplification in XPA/p53 transgenic mice exposed to Zidovudine (AZT). Toxicologist 103:344 (2009).
Olivero OA: Mechanisms of genotoxicity of nucleoside reverse transcriptase inhibitors. Environ Mol Mutagen 48:215-223 (2007).

Pedeutour F, Suijkerbuijk RF, Forus A, Van Gaal J, Van de Klundert W, et al: Complex composition and co-amplification of SAS and MDM2 in ring and giant rod marker chromosomes in well-differentiated liposarcoma. Genes Chromosomes Cancer 10:85-94 (1994).

Pinkel D, Straume T, Gray JW: Cytogenetic analysis using quantitative, high-sensitivity, fluorescence hybridization. Proc Natl Acad Sci USA 83:2934-2938 (1986).

Shimizu N, Itoh N, Utiyama H, Wahl GM: Selective entrapment of extrachromosomally amplified DNA by nuclear budding and micronucleation during S phase. J Cell Biol 140: 1307-1320 (1998).

Shimizu N, Shimura T, Tanaka T: Selective elimination of acentric double minutes from cancer cells through the extrusion of micronuclei. Mutat Res 448:81-90 (2000)
Suijkerbuijk RF, Olde Weghuis DE, Van den Berg M, Pedeutour F, Forus A, et al: Comparative genomic hybridization as a tool to define two distinct chromosome 12-derived amplification units in well-differentiated liposarcomas. Genes Chromosomes Cancer 9:292295 (1994).

van Steeg H, de Vries A, van Oostrom CT, van Benthem J, Beems RB, van Kreijl CF: DNA repair-deficient $X p a$ and $X p a / p 53+/$ - knockout mice: nature of the models. Toxicol Pathol 29 Suppl:109-116 (2001).

Wang X, Thomas P, Xue J, Fenech M: Folate deficiency induces aneuploidy in human lymphocytes in vitro-evidence using cytokinesis-blocked cells and probes specific for chromosomes 17 and 21. Mutat Res 551:167180 (2004).

Xu GL, Bestor TH, Bourc'his D, Hsieh CL, Tommerup N, et al: Chromosome instability and immunodeficiency syndrome caused by mutations in a DNA methyltransferase gene. Nature 402:187-191 (1999).

Yu M, Ward Y, Poirier MC, Olivero OA: Centrosome amplification induced by the antiretroviral nucleoside reverse transcriptase inhibitors lamivudine, stavudine, and didanosine. Environ Mol Mutagen 50:718-724 (2009). 